AperTO - Archivio Istituzionale Open Access dell'Università di Torino

\title{
Solid lipid nanoparticles and microemulsions for drug delivery The CNS.
}

\section{This is the author's manuscript}

Original Citation:

\section{Availability:}

This version is available http://hdl.handle.net/2318/75935

since

Published version:

DOI:10.1016/S0079-6123(08)80010-6

Terms of use:

Open Access

Anyone can freely access the full text of works made available as "Open Access". Works made available under a Creative Commons license can be used according to the terms and conditions of said license. Use of all other works requires consent of the right holder (author or publisher) if not exempted from copyright protection by the applicable law. 
H.S. Sharma (Ed.)

Progress in Brain Research, Vol. 180

ISSN: 0079-6123

Copyright @ 2009 Elsevier B.V. All rights reserved.

\title{
Solid lipid nanoparticles and microemulsions for drug delivery: the CNS
}

\author{
Maria Rosa Gasco ${ }^{1}$, Lorenzo Priano ${ }^{2,3}$ and Gian Paolo Zara ${ }^{4}$ \\ ${ }^{1}$ Nanovector s.r.l, Torino, Italy \\ ${ }^{2}$ Department of Neurosciences, University of Turin, Torino, Italy \\ ${ }^{3}$ IRCCS - Istituto Auxologico Italiano, Ospedale S. Giuseppe - Piancavallo, Oggebbio, Italy \\ ${ }^{4}$ Department of Anatomy Pharmacology and Forensic Medicine, University of Turin, Torino, Italy
}

\begin{abstract}
The chapter examined solid lipid nanoparticles (SLN) and microemulsions, chosen as carriers of drugs, administered in vivo to be transported to the central nervous system. Drugs of different structures and for different therapies have been studied such as doxorubicin SLN stealth and nonstealth administered in rats by intravenous route, apomorphine SLN administered in rats by duodenal route, melatonin SLN administered by transdermal and oral routes in humans, and apomorphine microemulsion administered by transdermal route in Parkinson's patients. The pharmacokinetics of the drug, followed in most studies, put in evidence that the many important pharmacokinetic parameters were notably improved versus the drug alone or in a commercial formulation.
\end{abstract}

Keywords: solid lipid nanoparticles; microemulsions; drug delivery system; central nervous system

\section{Introduction}

The brain homeostasis is of primary importance for survival so that specific interfaces, also referred to as barriers, tightly regulate the exchange between the peripheral blood circulation and the cerebrospinal fluid (CSF) circulatory system. These barriers are represented by the choroid plexus epithelium, the arachnoid epithelium, and the blood-brain barrier (BBB). The concentration and clearance of endogenous and exogenous molecules, essential for the normal brain functions or dangerous because of their toxicity, are strictly regulated by the anatomic and physiologic features of each barrier (Abbott, 2002; Segal, 2000).
The presence of the BBB is certainly the most critical issue encountered in brain drug delivery. Among the possible strategies to deliver therapeutic molecules into the brain, namely, intracerebral, intraventricular, and intravascular delivery, the latest represents the most reliable one because of its potential efficacy, safety, and compliance (Silva, 2007).

Brain capillaries, differently from the peripheral capillaries, present no fenestrae, a low amount of pinocytosis vesicles and particular tight junctions also known zonula occludens. Tight junctions are structures that form a narrow and continuous seal surrounding each endothelial and epithelial cell at the apical border and are at strictly regulating the movements the molecules through the paracellular 
01 pathway. These structures, together with the brain 02 endothelial cells, make an almost impermeable 03 barrier for drugs administered through the peripheral circulation (Kniesel \& Wolburg, 2000; Lapierre, 2000).

A further contribution to the peculiar BBB 7 functions is given by the periendothelial structures represented by astrocytes, pericytes, and the basal membrane (Balahanov \& Dore-Duffy, 1998; Lay \& Kuo, 2005).

The presence of BBB transport systems further 12 complicates the scenario. In fact, these transpor3 ters may assist or hinder the drug delivery to the ${ }_{4}$ brain. The carrier-mediated transport may be able to shuttle drugs or prodrugs into the brain in 6 therapeutic concentrations, mimicking nutrients 7 or endogenous compounds (Conford \& Hyman, 1999; Pardrige, 1998).

Unfortunately, the presence of active efflux transporters to the $\mathrm{BBB}$ also limits the therapeutic 1 efficacy of drugs virtually able to access the brain. 2 The P-glycoprotein (P-gp) is an ATP-dependent drug transport protein present at the apical mem24 branes of different epithelial cell types including those forming the BBB.

Recently, it has been demonstrated, either in vitro or in vivo, that BBB P-gp can prevent the 28 accumulation of many molecules including a variety of drugs in the brain (Stouch \& Gudmundsson, 2002), and P-gp inhibition has been proposed as a 1 possible strategy to enhance the drug penetration (Skinkel, 1999).

Different strategies have been studied for the ${ }_{4}$ delivery of drugs to the brain. Indeed most part of 35 the small drug molecules and of large molecules ${ }_{6}$ such as recombinant proteins or gene-based mole7 cules are not able to penetrate the BBB and many efforts have been spent in the previous years toward delivery and targeting of drugs to the brain (de Boer \& Gaillard, 2007). Many investigations have been carried out in the previous years 2 to improve brain tumors therapy with nanoparticulates; there are less number of studies regarding colloidal carriers of drugs for neurological diseases 4 or of diagnostics. Liposomes, polymeric nanopar46 ticles, and solid lipid nanoparticles (SLN) have 4 been studied, with different approaches, and the ${ }_{48}$ problems of overcoming the BBB.
In this chapter, we consider SLN and microemulsions as carriers for the delivery only of drugs active on the central nervous system (CNS). In particular, examining drugs used for therapy in neurological diseases, as many times their administration gives problems, such as high amount of drug administered by parenteral route, short halflife, high hydrophilicity, and poor transport through the BBB. The aim of all the researchers is to study if some improvements in pharmacokinetic parameters in laboratory animals and/or in humans could be achieved using colloidal formulations; the review considers studies on SLN and microemulsions carrying only drugs active on CNS.

\section{Solid lipid nanoparticles}

Different approaches are followed for the SLN preparation.

They can be prepared by high-pressure homogenization at elevated or low temperatures, via warm microemulsions, by solvent emulsificationevaporation-diffusion, by high-speed stirring, and/ or sonication (Muller, Kader, \& Gohla, 2000).

Here we refer only about SLN carrying drugs active on CNS (at brain level).

SLN carrying the lipophilic antipsychotic drug clozapine were prepared by hot homogenization followed by ultrasonication method. Clozapine has a very poor bioavailability (Manjunath \& Venkateswarlu, 2005). The SLN were administered by intravenous (IV) and duodenal routes to Swiss albino mice. For the intravenous administration, stearylamine was entrapped with clozapine in SLN; the area under curve (AUC) in the brain increased up to 2.91-fold the one of clozapine suspension.

The same authors (Manjunath \& Venkateswarlu, 2006) developed SLN as carriers of the highly lipophilic drug nitrendipine, using different triglycerides for the lipid matrix, soy lecithin, and Poloxamer 188. Positive and negative charged nitrendipine SLN were also produced and then examined to explore the influence of the charge on oral bioavailability. The different kinds of SLN were administered by IV and intraduodenal 
01 homogenized. The SLN were labelled with ${ }^{3} \mathrm{H}$ cetyl alcohol. The transport of the nanoparticles was measured by an "in situ" rat brain perfusion method; significant uptake of SLN was obtained . suggesting CNS uptake. The same group studied also the effect that the addition of a thiamine ligand to NPs, obtained by microemulsion as precursors, causes association with the BBB thiamine transporter (Lockman et al., 2003).

Muller and coworkers studied the preferential adsorption of blood protein onto intravenously injected particulate carriers from different origins (Luck, Paulke, Schroder, Blunk, \& Muller, 1998); in particular, Apolipoprotein E (Apo E) on the surface of P80-coated SLN after their incubation in human plasma citrate. Delivery to the brain using nanoparticulate drug carriers in combination with the targeting principles of "differential protein adsorption" has been proposed (Dehouck et al., 1997). The Pathfinder technology (Muller \& Schmidt, 2002) exploits proteins present in the blood which absorb onto the surface of intravenously injected carriers for targeting nanoparticles to the brain. Apo E is one of such targeting molecules for the delivery of nanoparticles to the endothelial cells of the BBB. Apo E can play an important role in the transport of lipoprotein into brain via the low-density lipoprotein receptor present on the BBB. Atoquavone (Muller \& Keck, 2004; Scholler et al., 2001) is a drug poorly adsorbed after oral administration, showing poor therapeutic efficacy against toxoplasma encephalitis (TE). Nanocrystals of the drug were produced, their surface was modified with Tween 80 leading to in vivo preferential absorption of Apo $\mathrm{E}$; the nanosuspension was IV administered to a murine model of TE, obtaining the disappearance of parasites and of cysts at dose 10-fold smaller than the one of atoquavone administered by oral route.

\section{Solid lipid nanoparticles from warm microemulsions}

SLN can be achieved from warm microemulsions.

Warm microemulsions are prepared at temperature ranging from $60^{\circ} \mathrm{C}$ to $80^{\circ} \mathrm{C}$ by using AU7 
01 melted lipids (such as triglycerides/fatty acids) as

02 oil, surfactants such as lecithin, and cosurfactants

AU8 03 (such as short-chain carboxylates, biliar salts); the 04 warm microemulsions are subsequently dispersed 05 in cold water. The nanodroplets of warm micro${ }_{06}$ emulsion, using this procedure, become SLN; they 07 are successively washed by tangential flow filtra08 tion. SLN are spherical in shape and with a narrow 09 size distribution. The zeta potential is normally 10 high $(30 / 40 \mathrm{mV})$ being positive or negative 11 depending on the starting formulation.

12 Hydrophilic and lipophilic molecules (drugs or 13 diagnostics) can be incorporated in SLN using 14 different methods.

SLN are able to carry drugs of different structure and lipophilicity, such as cyclosporine A 17 (Ugazio, Cavalli, \& Gasco, 2002), paclitaxel 18 (Cavalli, Caputo, \& Gasco, 2000), doxorubicin 19 (Fundaro, Cavalli, Bargoni, Vighetto, \& Gasco, 20 2000), tobramycin (Cavalli et al., 2003), short-

AU9 21 chain fatty acids (Dianzani et al., 2006), peptides ${ }_{22}$ (Morel, Cavalli, \& Gasco, 1996), antisense oligo23 nucleotides (Brioschi et al., 2008), and melatonin 24 (MT) (Rezzani et al., 2009). Also diagnostic compounds such as iron oxides (Pereira, 2003) have been incorporated into SLN.

SLN can be internalized within 2-3 min into all 8 the tested cell lines (Miglietta, Cavalli, Bocca, Gabriel, \& Gasco, 2000; Serpe et al., 2006); administered by duodenal route and are targeted to lymph (Bargoni et al., 1998). SLN stealth can 32 also be prepared to avoid their recognition by 3 the RES, thus prolonging their residence time (Podio, 2001). SLN drug, unloaded or loaded, stealth/or nonstealth, are transported through the BBB (Podio, 2001; Zara et al., 2002).

\section{Drug-loaded solid lipid nanoparticles}

40

${ }^{41}$ In the late 1990s SLN were proposed for brain

${ }^{42}$ drug targeting by several groups (Yang, Zhu, Lu,

${ }^{43}$ \& Liang, 1999; Zara et al., 1999), which studied

${ }^{44}$ the pharmacokinetics of two anticancer agents:

${ }^{45}$ camptothecin and doxorubicin. After oral and IV

${ }^{46}$ administration, they observed drug accumulation

47 into the brain.
Both stealth and nonstealth stearic acid unloaded labelled SLN were found in rat CSF 20 min after IV administration even though low amount of radioactivity was found in the CSF samples collected from cysterna magna (Podio, Zara, Carazzone, Cavalli, \& Gasco, 2000).

When the same kind of SLN were loaded with doxorubicin, significantly higher drug concentrations were found in the brain of the animals treated with stealth SLN as compared to nonstealth SLN and doxorubicin solution. The overall plasma pharmacokinetics of stealth and nonstealth SLN provided to be significantly different from that of the doxorubicin solution (Fundaro et al., 2000).

$R$-apomorphine (10,11-dihydroxyapomorphine) is a well-known potent short-acting dopamine agonist at D1 and D2 dopamine receptors and it was proposed as an antiparkinsonian drug more than a century ago. It significantly reduces the severity and duration of "off" periods and it is able to reverse bradykinesia when administered alone. Despite these favorable clinical effects, the drug's clinical use is somewhat limited by its pharmacokinetic profile: short half-life $(\sim 30 \mathrm{~min})$, rapid clearance from the plasma, lack of storage and retention in brain regions, poor oral bioavailability $(5 \%)$, and first-pass hepatic metabolism are significant limitations to chronic oral administration. Our group evaluated a new formulation of apomorphine in SLN (submitted data for publishing); the study was designed to investigate the AU12 pharmacokinetics and biodistribution of apomorphine incorporated in SLN, injected orally or intravenously in rats.

In vitro the release over time of apomorphine from the SLN dispersion was almost linear After IV administration the peak plasma concentration was higher after apomorphine solution administration than after apomorphine SLN. However, the total area under curve $\left(\mathrm{AUC}_{\text {tot }}\right)$ was nonsignificantly different after SLN than apomorphine solution. The terminal half-life was significantly longer following apomorphine SLN.

Following intraduodenal administration we found that the $C_{\max }$ and $\mathrm{AUC}_{\text {tot }}$ were significantly higher with apomorphine SLN compared to apomorphine solution; on the contrary, the clearance 
$$
\text { (n) }
$$

$$
28
$$$$
\text { (n) }
$$$$
\begin{aligned}
& 30 \\
& 31
\end{aligned}
$$$$
32
$$$$
33
$$$$
34
$$$$
35
$$$$
36
$$$$
37
$$$$
38
$$$$
39
$$$$
40
$$$$
41
$$$$
42
$$$$
43
$$$$
44
$$$$
45
$$$$
46
$$$$
47
$$

48 . Plasma levels of fre

apomorphine SLN in rats. incorporated MT in SLN (MT-SLN). MT has been chosen for our in vivo study because of its safeness in humans even at high dosages.

MT is a hormone produced by the pineal gland at night, involved in the regulation of circadian rhythms. For clinical purposes (mainly disorders of the sleep-wake cycle and insomnia in the elderly), exogenous MT administration should mimic the typical nocturnal endogenous MT levels, but its pharmacokinetics is not favorable due to its short half-life of elimination (DeMuro, Nafziger, Blask, Menhinick, \& Bertino, 2000; Mallo et al., 1990). The pharmacokinetics of MTSLN has been examined in humans after administration by oral and transdermal route (Priano et al., 2007). Three kinds of freeze-dried MTSLN containing different amounts of MT were prepared and characterized: (a) MT-SLN: MT = $1.8 \%$ for in vitro experiments (average diameter: $85 \mathrm{~nm}$, polydispersity index $=0.135$ ); (b) MT-SLN: $\mathrm{MT}=2 \%$ for transdermal application (average diameter $=91 \mathrm{~nm}$, polydispersity index $=0.140$ ); and (c) MT-SLN: MT $=4.13 \%$ for oral route (average diameter $=111 \mathrm{~nm}$, polydispersity index $=0.189$ ).

In vitro, MT-SLN produced a flux of MT of 1 $\mu \mathrm{g} / \mathrm{h} / \mathrm{cm}^{2}$ through hairless mice skin, following a pseudo-zero-order kinetics (45). At the same time, AU13 in vivo study produced very interesting results,

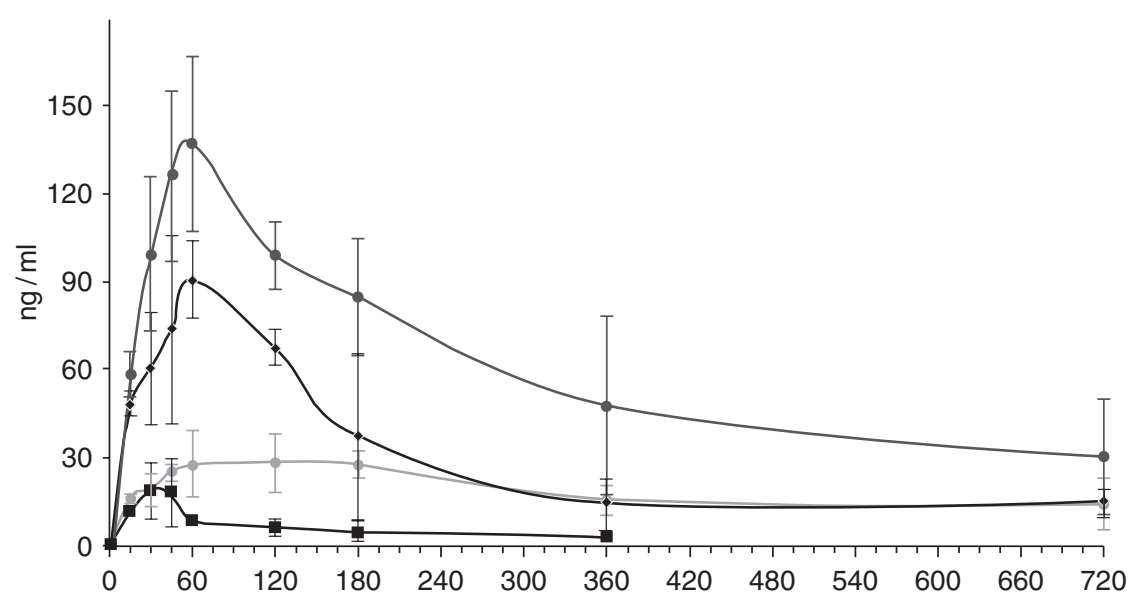


01 confirming in humans that SLN can act as a reser02 voir that allows a constant and prolonged release ${ }_{03}$ of the included drugs (Peira et al., 2003). MT ${ }_{04}(3 \mathrm{mg})$ incorporated in SLN was orally adminis${ }_{05}$ tered at 8.30 a.m. to seven healthy subjects; for o6 control purposes, 1 week later the same subjects 07 received orally a standard formulation of MT at 08 the same dose $(3 \mathrm{mg})$ and again at 8.30 a.m. Compared to the MT standard solution, $T_{\max }$ observed after MT-SLN administration was delayed of 1 about 20 min, while mean AUC and mean half12 life of elimination were significantly higher (respectively $169,944.7 \pm 64,954.4 \mathrm{pg} / \mathrm{mL} \times$ hour 4 vs. $85,148.4 \pm 50,642.6 \mathrm{pg} / \mathrm{mL} \times$ hour, $p=0.018$; 15 and $93.1 \pm 37.1 \mathrm{~min}$ vs. $48.2 \pm 8.9 \mathrm{~min}, p=0.009$ ). 16 Even more, standard formulation and MT-SLN 17 after oral administration produced similar peak s plasma levels of MT, even if delayed of about 9 half an hour in the case of MT-SLN. More inter20 estingly, detectable and clinically significant MT plasma levels after MT-SLN oral administration 2 were maintained for a longer period of time, suggesting that SLN orally administered to humans ${ }_{24}$ can yield a sustained release of the incorporated drug, a feature that could be particularly useful for molecules, such as MT, characterized by unfavorable kinetics (Priano et al., 2007). Previous studies in laboratory animals indicated a probable targeting of SLN - either drug-loaded or unloaded to lymph, after duodenal administration (Bargoni et al., 1998). Similarly, the significantly longer half-life of MT observed in the study of Priano et al. (2007) may suggest a targeting of MT-SLN to human lymph, even though the capsules used to administer SLN were not gastro-resistant. In fact, MT half-life of elimination has been calculated in about $40 \mathrm{~min}$ after an intravenous bolus and following oral administration low bioavailability and rapid clearance from plasma have been shown, primarily due to a marked first-pass hepatic metabolism. Moreover, pharmacokinetic analysis following transdermal administration of MT-SLN demonstrated that plasma levels of MT similar to those produced by oral administration may be achieved for more than $24 \mathrm{~h}$ (50). In 10 healthy subjects, SLN incorporating MT were administered transdermally by applying a patch at 8.30 a.m. and leaving it in place for $24 \mathrm{~h}$. In this delivery system, MT absorption and elimination were slow (mean half-life of absorption $=5.3 \pm 1.3 \mathrm{~h}$; mean half-life of elimination $=24.6 \pm 12.0 \mathrm{~h}$ ) so that MT plasma levels above $50 \mathrm{pg} / \mathrm{mL}$ were maintained for at least $24 \mathrm{~h}$ (Figs. 2 and 3). Tolerability of MT-SLN administered transdermally or by oral

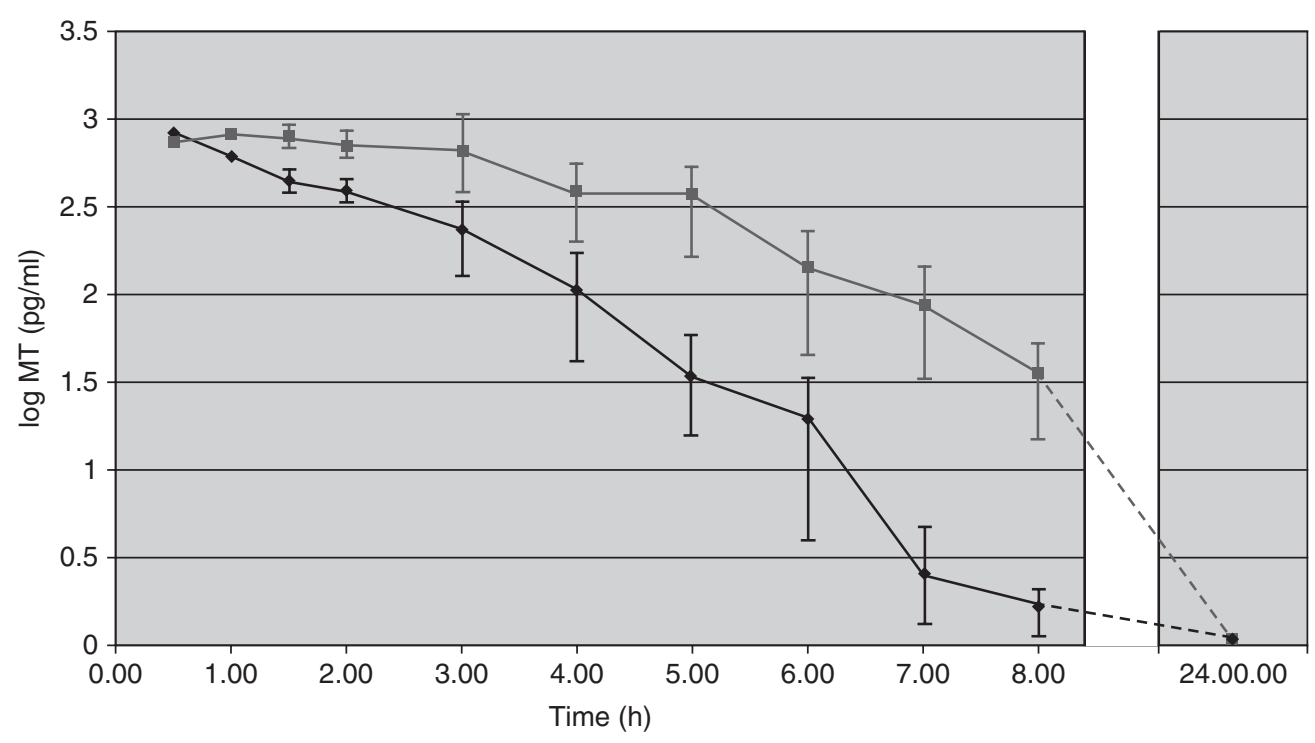

Fig. 2. MT plasma profile in humans after MT $(\bullet)$ and MT-SLN $(\bullet)$ oral administration. 

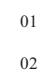

03

04

05

06

07

08

09

10

11

12

13

14

15

16

17 Fig. 3. MT plasma levels in humans at baseline and after MT-

18 SLN transdermal administration (MT-SLN-TD).

route was good and no adverse effect occurred, apart from a predictable mild somnolence and transient erythema after gel application. This means that, at least at the doses used in that study (45), SLN administration via the oral or transdermal routes is safe.

In this context, we also tested transdermal MTSLN for three consecutive nights in five patients suffering from delayed sleep phase syndrome (unpublished data), confirming the safeness of this formulation. Due to the small sample, however, the tendency of clinical benefits was present but statistical significance could not be reached, so that further investigations in larger samples are needed in order to evaluate the impact of this new formulation in clinical practice.

However, these very favorable results, obtained in humans administering MT-loaded SLN, clearly suggest that SLN can be considered effective in vivo delivery systems that could be suitably applied to different drugs, and in particular to those requiring prolonged high plasma levels but that have unfavorable pharmacokinetics. Finally, it must be stressed that, since doses and concentrations of drugs included in SLN can be varied, different plasma level profiles could be obtained, thus disclosing new chances for sustained delivery systems adaptable to a variety of clinical conditions (Priano et al., 2007).
Suitability of SLN to convey drugs into CNS is also confirmed by studies regarding baclofen included in SLN. Intrathecal baclofen administration represents the reference treatment for spasticity of spinal or cerebral origin. Nevertheless, surgical involvement together with risk of infection or catheter dysfunction may limit the number of potentially treatable patients (Dario \& Tomei, 2004; Perot \& Almeida-Silveira, 1994). In order to explore alternative and efficacious routes of administration, we studied a new pharmaceutical preparation characterized by SLN incorporating baclofen (baclofen-SLN) (submitted data for publishing). Baclofen concentration, after reconstitu- AU14 tion with water of freeze-dried SLN, was $1.7 \mathrm{mg}$ / $\mathrm{mL}$. Groups of Wistar rats were injected intraperitoneally with physiological solution and unloaded SLN at $10 \mathrm{~mL} / \mathrm{kg}$ (control groups), with baclofenSLN (baclofen-SLN group), and baclofen solution (baclofen-sol group) at increasing dosages of 2.5, $5,7.5,8.5$, and $10 \mathrm{mg} / \mathrm{kg}$. At different times up to the fourth hour, efficacy testing was performed by means of H-reflex, while behavioral characterization was obtained using two scales validated for motor symptoms due to spinal lesions and sedation in rat models (Nemethy, Paroli, WilliamsRusso, \& Blanck, 2002; Tsunoda, Kuang, Tolley, Whitton, \& Fujinami, 1998). Rats were sacrificed for detecting baclofen concentration in blood and tissue. Compared to baclofen-sol and control group, $H / M$ amplitude curve after baclofen-SLN injection was characterized by a dose-dependent reduction at the first and second hours, so confirming efficacy, and a rebound increase at the fourth hour, indicating an unexpected belated spinal hyperexcitability (Fig. 4). Similarly, baclofen-SLN effect on behavioral scales was stronger compared to baclofen-sol group, with the maximum effects obtained at the first hour. Moreover, clinical effects were detectable after low dosages of baclofen-SLN $(2.5 \mathrm{mg} / \mathrm{kg})$ but only after higher dosages of baclofen-sol $(7.5 \mathrm{mg} / \mathrm{kg})$. After $4 \mathrm{~h}$ from the injection, only the rats treated with the higher dosages of baclofen-SLN still presented clinical signs consisting in sedation $(8.5 \mathrm{mg} / \mathrm{kg})$ or complete paralysis and piloerection $(10 \mathrm{mg} / \mathrm{kg})$. On the whole, these data suggest a dose-dependent modulation of spinal reflex excitability, which is 
${ }^{28}$ baclofen-sol. In brain, both the two formulations

${ }^{29}$ (baclofen in solution and in SLN) gave a maxi-

${ }^{30}$ mum after $2 \mathrm{~h}$ but concentrations after SLN were

${ }^{31}$ almost twice the ones after solution. This last data

${ }^{32}$ might be due partly to the free drug already

${ }^{33}$ released and to baclofen-SLN overcoming the

${ }^{34} \mathrm{BBB}$. We realize that for clinical purposes this

AU15 35 effect of baclofen-SLN is unwished, as it is respon-

${ }^{36}$ sible for sedation. However, baclofen-sol injec-

${ }^{37}$ tions also produced sedation, even if weaker and

${ }^{38}$ corresponding to lower plasma concentrations,

39 compared to baclofen-SLN. In conclusion, higher

${ }^{40}$ spinal and cortical effects of baclofen-SLN, com-

${ }^{41}$ pared to equivalent dosages of baclofen-sol, seem

${ }^{42}$ attributable to higher and more prolonged con-

${ }^{43}$ centrations of drugs in plasma and brain.

${ }_{44}$ As previously noted, unloaded SLN adminis-

${ }^{45}$ tered by duodenal route are targeted to lymph

${ }^{46}$ and the incorporated drug can be partly distribu-

${ }^{47}$ ted in the brain; moreover, SLN can also be

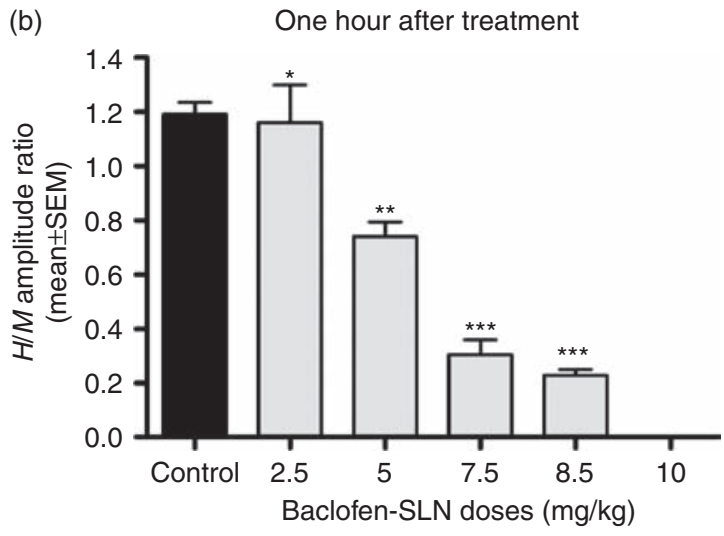

prepared stealth for increasing their residence time (Bargoni et al., 1998; Fundaro et al., 2000; Podio, 2001; Zara et al., 2002). Other new studies will be directed toward a duodenal administration of baclofen-SLN stealth, not only for prolonging their residence time but also to target them to lymph, enhancing their bioavailability. Further research should also be directed toward the optimization of dosages and concentrations of baclofen included in SLN, in order to preserve the prolonged antispastic effect, peculiar of this new formulation, but devoid of clinically significant cortical effects.

\section{Solid lipid nanoparticles as potential diagnostics}

Superparamagnetic iron oxides are classified as contrast agents for magnetic resonance imaging (MRI). They are able to affect the water relaxation times $T_{1}$ and $T_{2}$; their ability in altering such properties is quantified by the parameter relaxivity. Iron oxides are able to affect preferentially the $T_{2}$ relaxation times of tissues (and are called $T_{2}$-relaxing agents) while paramagnetic contrast agents such as $\mathrm{Gd}$ complexes affect mainly $T_{1}$ and are called $T_{1}$-relaxing agents.

Iron oxides are insoluble in water; therefore, to be clinically used they must be transformed in modified colloids while their magnetic properties 
01 should remain unchanged. The surface of the iron oxide nanoparticles can be modified, covering them by hydrophilic macromolecules; such as dextran in the case of Endorem.

A research was performed in order to know whether SLN can load iron oxides and whether they are able to reach the brain. Two different SLN, SLN-Fe ${ }^{\mathrm{A}}$ and $\mathrm{SLN}^{\mathrm{B}}$ containing iron oxides were prepared from warm microemulsions and studied at first in vitro (1). The comparison of Fe-SLN was performed with Endorem. Both the Fe-SLN preparations showed relaxometric properties similar to the ones of Endorem. The good $T_{2}$-relaxation-enhancing properties allow an in vivo study of their distribution by MRI. Fe$\mathrm{SLN}^{\mathrm{B}}$, at the higher $\mathrm{Fe}$ concentration, were administered IV to rats; the comparison was performed with Endorem. Images obtained after Endorem IV administration show early modification, but soon return to baseline; these findings are consistent with short Endorem retention time in blood. Results from SLN-Fe ${ }^{B}$ show a different behavior. For each part of the brain, maximal SS is reached in the last images (135 min after administration). SS increase from the first to the last acquisition. This study shows that after inclusion in SLN, Endorem becomes a new type of contrast agent: Endorem is taken by the liver and does not cross the BBB, while Endorem containing SLN-Fe ${ }^{\mathrm{B}}$ shows CNS uptake. This means that SLN-Fe kinesis is related to SLN and not to their iron oxide content as already seen.

\section{Microemulsions}

Microemulsions are transparent, thermodynamically stable dispersions of water and oil, usually stabilized by a surfactant and a cosurfactant. They contain particles smaller than $0.1 \mu \mathrm{m}$. Microemulsions are often defined as thermodynamically stable liquid solutions; the stability of microemulsions is a consequence of the ultralow interfacial tension between the oil and water phases. A clear distinction exists between microemulsion and coarse emulsions. The latter are thermodynamically unstable, droplets of their dispersed phase are generally larger than $0.1 \mu \mathrm{m}$ and consequently their appearance is normally milky rather than transparent.

The limits in the use of microemulsions in the pharmaceutical field are chiefly from the need of all the components to be acceptable, particularly surfactants and cosurfactants - the amounts of surfactants and cosurfactants required to form microemulsions are usually higher than those required for emulsions.

Recently, apomorphine was incorporated into microemulsions to study whether they are a feasible vehicle for transdermal transport of this drug. In the preparatory in vitro study (Peira, Scolari, \& Gasco, 2001), two different microemulsions whose components were all biocompatible were studied: the concentration of apomorphine was $3.9 \%$ in each. Since apomorphine is highly hydrophilic, to increase its lipophilicity, apomorphine-octanoic acid ion pairs were formed. At $\mathrm{pH} 6.0, \log P_{\text {app }}$ of apomorphine increased from 0.3 in the absence of octanoic acid to $\log P_{\text {app }}=2.77$ for a molar ratio 1:2.5 (apomorphine: octanoic acid). The flux of drug from the two thickened microemulsions through hairless mouse skin was, respectively, 100 and $88 \mu \mathrm{g} / \mathrm{h} / \mathrm{cm}^{2}$. The first formulation, having the higher flux, was chosen for in vivo administration to Parkinson's patients.

For the in vivo study, 21 patients with idiopathic Parkinson's disease who presented long-term L-DOPA syndrome, motor fluctuations and prolonged "off" periods were selected (Priano et al., 2004). Here, $10 \mathrm{~g}$ of apomorphine hydrochloride $(3.9 \%)$, included in microemulsion for transdermal delivery (Apo-MTD), was applied to a 100 $\mathrm{cm}^{2}$ skin area on the chest; the area was delimited by 1-mm-thick biocompatible foam tape and covered with a polyester-based membrane and an occlusive membrane to prevent evaporation. In these conditions, a single layer of microemulsion ( $1 \mathrm{~mm}$ thick) was directly in contact with the skin surface and acted as a reservoir of apomorphine. Apo-MTD was applied at 8.00 a.m. and left for 12 h. In all patients, except two, apomorphine was detected in blood samples after a variable lag time. Pharmacokinetic analysis revealed that epicutaneous-transdermal apomorphine absorption was rapid (mean half-life of absorption $=1.03 \mathrm{~h}$ ) 
01 with a variability among patients (half-life of ${ }_{02}$ absorption, $\mathrm{SD}=1.39 \mathrm{~h}$ ). Mean $C_{\max }$ was above ${ }_{03}$ the therapeutic range (mean $C_{\max }=42.81 \pm 11.67$ ${ }_{04} \mathrm{ng} / \mathrm{mL}$ ), with a mean $T_{\max }$ of $5.1 \pm 2.24 \mathrm{~h}$. Theros apeutic concentrations of apomorphine were o6 reached after a mean latency of $45 \mathrm{~min}$ (range ${ }_{07}$ 18-125), and stable concentrations, above the os therapeutic range, continued for as long as Apo09 MTD was maintained in place. At the 12th hour, 10 Apo-MTD was removed, and the apomorphine 11 plasma concentration then decreased at a rate 12 comparable to that described for subcutaneous 13 administration (mean half-life of elimination 4 equal to $10.8 \pm 1.93 \mathrm{~h})$. $C_{\max }$ and AUC showed 15 good correlations with the reduction of "off" periods duration and with the improvement of clinical scores evaluating motor performances ( $r$ values ranging form 0.49 to 0.56 , with $p$ values ranging 9 from 0.02 to 0.04 ). Apo-MTD overall tolerability was good: systemic side effects were similar to those caused by subcutaneous apomorphine injec2 tion (sleepiness, mild orthostatic hypotension, and 3 transient nausea), and in the case of nausea, they were strictly related to the highest plasma level of s apomorphine. Moreover, regarding local side 26 effects, the large majority of patients $(71.4 \%)$ presented a transient mild erythema at the site of Apo-MTD application, with a complete regression within $48 \mathrm{~h}$, whereas only in two cases the erythema lasted more than 3 days and required local therapy. This study clearly demonstrated 2 that in most Parkinson's patients Apo-MTD is 3 absorbed by the epicutaneous-transdermal route. This result is in contrast with other reports, where 35 the transdermal route did not produce detectable 36 plasma levels of apomorphine, or in which no apomorphine was transported passively through the skin (Gancher, Nutt, \& Woodward, 1991; van der Geest, 9 van Laar, Gubbens-Stibbe, Boddé, \& Danhof, 1997). Probably, this difference was mainly due to the peculiar pharmaceutical preparation used. Even 42 if pharmacokinetic parameters are variable, Apo43 MTD demonstrated the feasibility of providing ther${ }_{44}$ apeutic apomorphine plasma levels for much longer ${ }_{45}$ periods of time than previously tested apomorphine ${ }_{46}$ preparations (several hours), allowing a more con${ }_{47}$ stant dopaminergic stimulation. These results are 48 encouraging and Apo-MTD might become of clinical value in some parkinsonian patients suffering from uncontrolled "wearing-off" and prolonged "off" phenomena. On the contrary, because of the lag time of about $1 \mathrm{~h}$ before therapeutic concentrations are reached, Apo-MTD may not be the "ideal" preparation for rapid relief of "off" periods.

Since Apo-MTD was found to provide constant drug release over several hours, other studies have been addressed to its use for the nocturnal sleep disorders of Parkinson's patients. Twelve parkinsonian patients underwent standard polysomnography on basal condition and during one night treatment with Apo-MTD (applied to $100 \mathrm{~cm}^{2}$ from 10 p.m. until 8 a.m.; Priano et al., 2003). Sleep analysis during APO-MTD treatment in comparison to basal condition showed very favorable findings: $16 \%$ increment of total sleep time, $12 \%$ increment of sleep efficiency, $16 \%$ increment of stage 3 and 4 nonrapid eye movement (NREM), 15\% reduction of periodic limb movements index, $22 \%$ reduction of arousal index, and $23 \%$ reduction of the "cycling alternating pattern" rate, an objective measure of disruption and fragmentation of NREM sleep. Pharmacokinetic analysis confirmed the absorption of apomorphine and the maintenance of therapeutic plasma levels for several hours (mean $C_{\max }=31.8 \pm 9.7 \mathrm{ng} / \mathrm{mL}$; mean $T_{\max }=3.1 \pm 1.6 \mathrm{~h}$; mean half-life of absorption $=1.2 \pm 1.4 \mathrm{~h}$; mean half-life of elimination $=$ $8.8 \pm 1.9 \mathrm{~h})$. On the whole, this study confirmed that APO-MTD in Parkinson's disease might be able to reduce nocturnal anomalous movements, akinesia, and rigidity, and might be efficacious for reducing the instability of sleep maintenance typical of parkinsonian sleep.

\section{References}

Abbott, N. J. (2002). Astrocyte-endothelial interactions and bloodbrain-barrier permeability. Journal of Anatomy, 200, 629-638.

Balahanov, R., \& Dore-Duffy, P. (1998). Role of the CNS microvascular pericyte in the blood-brain barrier. Journal of Neuroscience Research, 53, 637-644.

Bargoni, A., Cavalli, R., Caputo, O., Fundaro, A., Gasco, M. R., \& Zara, G. P. (1998). Solid lipid nanoparticles in lymph and plasma after duodenal administration to rats. Pharmaceutical Research, 15, 745-750.

Brioschi, A., Calderoni, S., Pradotto, L. G., Guido, M., Strada, A., Zenga, F., et al. (2008). Solid lipid nanoparticles 


$$
\text { " }
$$$$
\text { 323-355. }
$$

carrying oligonucleotides inhibit vascular endothelial grow factor expression in rat glioma models. Journal of Nanoneuroscience, 1, 1-10.

Cavalli, R., Caputo, O., \& Gasco, M. R. (2000). Preparation and characterization of solid lipid nanospheres containing paclitaxel. European Journal of Pharmaceutical Science, 10, 305-308.

Cavalli, R., Bargoni, A., Podio, V., Muntoni, E., Zara, G. P., \& Gasco, M. R. (2003). Duodenal administration of solid lipid nanoparticles loaded with different percentages of tobramycin. Journal of Pharmaceutical Sciences, 92, 1085-1094.

18 de Boer, A. G., \& Gaillard, P. J. (2007). Drug targeting to the

21 Dehouck, R., Ferrari, L., Dehouck, M. P., Pierce, A., Torpier, G., \& Cecchelli, R. (1997). A new function for the LDL receptor. Transcytosis of LDL across the blood brain barrier. Journal of Cell Biology, 38, 877-889.

24 DeMuro, R. L., Nafziger, A. N., Blask, D. E., Menhinick, A. M., \& Bertino, J. S. (2000). The absolute bioavailability of oral melatonin. Journal of Clinical Pharmacology, 40, 781-784.

${ }_{27}$ Dianzani, C., Cavalli, R., Zara, G. P., Gallicchio, M., Lombardi, G., Gasco, M. R., et al. (2006). Cholesteryl butyrate solid lipid nanoparticles inhibit adhesion of human neutrophils to endothelial cells. British Journal of Pharmacology, 148, 648-656.

30 Fundaro, A., Cavalli, R., Bargoni, A., Vighetto, G. P., \& Gasco, M. R. (2000). Non-stealth and stealth solid lipid nanoparticles (SLN) carrying doxorubicin: Pharmacokientics and tissue distribution after i.v. administration to rats. Pharmacological Research, 42, 337-343.

34 Gancher, S. T., Nutt, J. G., \& Woodward, W. R. (1991).

35 Absorption of apomorphine by various routes in parkinsonism. Movement Disorders, 6, 212-216.

37 Gupta, Y., Jain, A., \& Jain, S. K. (2007). Transferrin conjugate solid lipid nanoparticles for enhanced delivery of quinine dihydrochloride to the brain. Journal of Pharmacy and Pharmacology, 59, 935-940.

40 Kniesel, K., \& Wolburg, H. (2000). Tight junctions of the blood $41 \quad$ brain barrier. Cellular and Molecular Neurobiology, 20, 57-76. ${ }_{42}$ Koziara, J. M., Lockman, P. R., Allen, D. D., \& Mumper, R. J. (2003). In situ blood-brain barrier transport of nanoparticles. Pharmaceutical Research, 20, 1772-1777.

44 Lapierre, L. A. (2000). The molecular structure of the tight 45 junctions. Advanced Drug Delivery Reviews, 41, 255-264.

46 Lay, C. H., \& Kuo, K. H. (2005). The critical component to 47 establish in vitro BBB model: Pericyte. Brain Research 47 Reviews, 50, 258-265.
Lockman, P. R., Oyewumi, M. O., Koziara, J. M., Roder, K. E., Mumper, R. J., \& Allen D. D. (2003). Brain uptake of thiaminecoated nanoparticles. Journal of Controlled Release, 93, 271-282.

Luck, M., Paulke, B. R., Schroder, W., Blunk, T., \& Muller, R. H. (1998). Analysis of plasma protein adsorption on polymeric nanoparticles with different surface characteristics. Journal of Biomedical Materials Research, 39, 478-485.

Mallo, C., Zaidan, R., Galy, G., Vermeulen, E., Brun, J., Chazot, G., et al. (1990). Pharmacokinetics of melatonin in man after intravenous infusion and bolus injection. European Journal of Clinical Pharmacology, 38, 297-301.

Manjunath, K., \& Venkateswarlu, V. (2005). Pharmacokinetics, tissue distribution and bioavailability of clozapine solid lipid nanoparticles after intravenous and intraduodenal administration. Journal of Controlled Release, 107, 215-228.

Manjunath, K., \& Venkateswarlu, V. (2006). Pharmacokinetics, tissue distribution and bioavailability of nitrendipine solid nanoparticles after intravenous and intraduodenal administration. Journal of Drug Targeting, 14, 632-645.

Miglietta, A., Cavalli, R., Bocca, C., Gabriel, L., \& Gasco, M. R. (2000). Cellular uptake and cytotoxicity of solid lipid nanospheres (SLN) incorporating doxorubicin or paclitaxel. International Journal of Phamaceutics, 210, 61-67.

Morel, S., Cavalli, R., \& Gasco, M. R. (1996). Thymopentin in solid lipid nanoparticles. International Journal of Phamaceutics, 132, 259-262.

Muller, R. H., Kader, K., \& Gohla, S. (2000). Solid lipid nanoparticles (SLN) for controlled drug delivery - a review of the state of the art. European Journal of Pharmaceutics and Biopharmaceutics, 50, 161-177.

Muller, R. H., \& Schmidt, S. (2002). Path. Finder technology for the delivery of drugs to the brain. New Drugs, 2, 38-42.

Muller, R. H., \& Keck, C. M. (2004). Challenges and solutions for the delivery of biotech. Drugs - a review of drug nanocrystal technology and lipid nanoparticle. Journal of Biotechnology, 113, 151-170.

Nemethy, M., Paroli, L., Williams-Russo, P. G., \& Blanck, T. J. (2002).Assessing sedation with regional anesthesia: Interrater agreement on a modified Wilson sedation scale. Anesthesia and Analgesia, 94, 723-728.

Pardrige, W. M. (1998). CNS drug design based on principles of blood-brain barrier transport. Journal of Neurochemistry, 70, 1781-1792.

Peira, E., Scolari, P., \& Gasco, M. R. (2001). Transdermal permeation of apomorphine through hairless mouse skin from microemulsions. International Journal of Phamaceutics, 226, 47-51.

Peira, E., Marzola, P., Podio, V., Aime, S., Sbarbati, A., \& Gasco, M. R. (2003). In vitro and in vivo study of solid lipid nanoparticles loaded with superparamagnetic iron oxide. Journal of Drug Targeting, 11, 19-24.

Perot, C., \& Almeida-Silveira, M. I. (1994). The human H and $\mathrm{T}$ reflex methodologies applied to the rat. Journal of Neuroscience Methods, 51, 71-76.

Podio, V., Zara, G. P., Carazzone, R., Cavalli, R., \& Gasco, M. R. (2000). Biodistribution of stealth and non-stealth solid 
lipid nanoparticles after intravenous administration in rats. Journal of Pharmacy and Pharmacology, 52, 1057-1063.

Priano, L., Albani, G., Brioschi, A., Guastamacchia, G. Calderoni, S., Lopiano, L., et al. (2003). Nocturnal anomalous movement reduction and sleep microstructure analysis in parkinsonian patients during 1-night transdermal apomorphine treatment. Neurological Science, 24 207- 208.

Priano, L., Albani, G., Brioschi, A., Calderoni, S., Lopiano, L., Rizzone, M., et al. (2004). Transdermal apomorphine permeation from microemulsions: A new treatment in Parkinson's disease. Movement Disorders, 19, 937-942.

11 Priano, L., Esposti, D., Esposti, R., Castagna, G., De Medici, C., Fraschini, F., et al. (2007). Solid lipid nanoparticles incorporating melatonin as new model for sustained oral and transdermal delivery systems. Journal of Nanoscience and Nanotechnology, 7, 3596-3601.

15 Rezzani, R., Fabrizio Rodella, L., Fraschini, F., Gasco, M. R., Demartini, G., Musicanti, C., et al. (2009). Melatonin delivery in solid lipid nanoparticles: Prevention of cyclosporin A induced cardiac damage. Journal of Pineal Research, 46, 255-261.

18 Segal, M. B. (2000). The choroid plexuses and the barriers between the blood and the cerebrospinal fluid. Cellular and Molecular Neurobiology, 20, 183-196.

${ }_{21}$ Scholler, N., Krause, K., Kayser, O., Muller, R. H., Borner, K., Hahn, H., et al. (2001). Atovaquone nanosuspensions show excellent therapeutic effect in a new murine model of reactivated toxoplasmosis. Antimicrobial Agents and Chemotherapy, 45, 1771-1779.

25 Skinkel, A. H. (1999). P-glycoprotein, a gatekeeper in the blood-brain barrier. Advanced Drug Delivery Reviews, 36, 179-194.

Serpe, L., Cavalli, R., Gasco, M. R., Muntoni, E., Cavalli, R., Panzanelli, P., et al. (2006). Intracellular accumulation and cytotoxicity of doxorubicin with different pharmaceutical formulations in human cancer cells. Journal of Nanoscience and Nanotechnology, 6, 3062-3069.

Silva, A. S. (2007). Nanotechnology approaches for drug and small molecule delivery across the blood brain barrier. Surgical Neurology, 67, 113-116.

Stouch, T. R., \& Gudmundsson, O. (2002). Progress in understanding the structure-activity relationship pf P-glycoprotein. Advanced Drug Delivery Reviews, 54, 315-328.

Tsunoda, I., Kuang, L. Q., Tolley, N. D., Whitton, J. L., \& Fujinami, R. S. (1998). Enhancement of experimental allergic encephalomyelitis (EAE) by DNA immunization with myelin proteolipid protein (PLP) plasmid DNA. Journal of Neuropathology \& Experimental Neurology, 57, 758-767.

Ugazio, E., Cavalli, R., \& Gasco, M. R. (2002). Incorporation of cyclosporin A in solid lipid nanoparticles in solid lipid nanoparticles. International Journal of Phamaceutics, 241, 341-344.

van der Geest, R., van Laar, T., Gubbens-Stibbe, J. M., Boddé, H. E., \& Danhof, M. (1997). Iontophoretic delivery of R-apomorphine - II: An in vivo study in patients with Parkinson's disease. Pharmaceutical Research, 14, 1804-1810.

Yang, S. C., Zhu, J. B., Lu, Y., \& Liang, C. Z. (1999). Body distribution of camptothecin solid lipid nanoparticles after oral administration. Pharmaceutical Research, 751-757.

Zara, G. P., Cavalli, R., Fundaro, A., Bargoni, A., Caputo, O., \& Gasco, M. R. (1999). Pharmacokinetics of doxorubicin incorporated in solid lipid nanospheres (SLN). Pharmaceutical Research, 40, 281-286.

Zara, G. P., Cavalli, R., Bargoni, A., Fundaro, A., Vighitto, D., \& Gasco, M. R. (2002). Intravenous administration to rabbits of non-stealth and stealth doxorubicin loaded solid lipid nanoparticles at increasing concentration of stealth agent: Pharmacokinetics and distribution of doxorubicin in brain and in other tissues. Journal of Drug and Targeting, 10, $327-335$. 
1

052

063

074

085

096

107

118

9

10

11

12

\section{Contents}

Please check the insertion of the colon after "delivery:" in the chapter title.

Please indicate the corresponding author.

Please provide the full form of 'MRT'.

Please check throughout if the text "in confront" can be changed to "in contrast".

Please provide the full form of 'NP'.

Please check if the abbreviation of 'apolipoprotein E' is OK.

Please check if the unit for temperature is correct.

Please check if it is "biliar" or "bile"?

Please check the change of "short fatty acids" to "short-chain fatty acids".

Reference "Pereira, 2003" is not listed in the reference list. Please provide.

Reference "Podio, 2001" is not listed in the reference list. Please provide.

Please provide the reference for "submitted data for publishing".

Please check throughout the chapter the presence of numbers within the brackets, for example, "(45), (50), (1)". Can they be deleted or are they references?

Please provide the reference for "submitted data for publishing".

Please check if the word "unwished" can be changed to "undesirable/unwanted".

The compound "SLN" ${ }^{\mathrm{B}}$ " is not clear. Please check throughout. Is it "SLN-Fe" or "Fe-SLN".

Please provide the full form of 'SS'.

Please provide volume details for Reference "Yang et al. 1999". 